\title{
A Representation for \\ Shape Based on Peaks and Ridges in the Difference of \\ Low-Pass Transform
}

\author{
James L. Crowley and Alice C. Parker*
}

CMU-RI-TR-83-4

\author{
The Robotics Institute \\ Carnegie-Mellon University \\ Pittsburgh, Pennsylvania 15213 \\ *Department of Electrical Engineering-Systems \\ University of Southern California
}

May 1983

Copyright (C) 1983 Carnegie-Mellon University

This research was partially supported by The Robotics Institute, National Science Foundation Grant No. APR75-08154, and by Naval Electronics System Command (NELC) Grant No. N00039-79-Z-0169. 





\section{Abstract:}

This paper defines a multiple resolution representation for the two-dimensional gray-scale shapes in an image. This representation is constructed by detecting peaks and ridges in the Difference of Low Pass (DOLP) transform. Descriptions of shapes which are encoded in this representation may be matched efficiently despite changes in size, orientation or position.

Motivations for a multiple resolution representation are prosented first, followed by the definition of the DOLP T'ransform. Techniques are then presented for encoding a symbolic structural description of forms from the DOL.P transform. This process involves detecting local peaks and ridges in each band-pass image and in the entire three-dimensional space defined by the DOIP transform. Linking adjacent peaks in different band-pass images gives a multiple resolution tree which describes shape. Peaks which are local maxima in this tree provido landmarks for aligning, manipulating, and matching shapes. Detecting and linking the ridges in each DOLP band-pass inage provides a graph which links peaks within a shape in a band-pass image and describes the positions of the boundaries of the shape at multiple resolutions. Detccting and linking the ridges in the DOLP three space describes clongated forms and links the largest peaks in the tree.

The principles for determining the correspondence between symbols in pairs of such descriptions are then described. Such correspondence matching is shown to be simplified by using the correspondence at lower resolutions to constrain the possible correspondence at higher resolutions. 


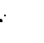




\section{Table of Conterts}

1 Introduction 1

1.1 Motivation:A Mulci-Resolution Structural Description of Inages

1.2 Propertics of the Representation 2

1.3 Correspondence Matching 4

1.4 Contencs of this Paper 5

2 The Difference of I ow-Pass Tiansform 5

2.1 The Purpose of the DOI.P Transform

2.2 Definition of the DOLP transform $\quad 6$

2.3 Fast Computacion Techniques: Resampling and Cascade Convolution 7

2.4 An Example: the DOI.P Transform of a Teapot Image 8

3 Construction of the Representation from a DOLP Transform 8

3.0.1 The Approach $\quad 11$

3.1 Detection of Pcak-Nodes and Ridge-Nodes within each Band-pass Image 12

3.2 Linking of Ridge-Paths at a Rand-Pass Level $\quad 13$

3.3 Linking Peaks Between Levels and Detecting the Largest Peak 16

3.4 Detecting the Largest Three-Dimensional Ridge Path 18

4 A Simple Exaniple of Watching $\quad 18$

4.1 Abstracting the Graph of Connected Peaks at a Level 19

4.1.1 Example of Abstracted P-nodes and R-paths $\quad 19$

4.2 Initial Alignment to Obtain Size and Position $\quad 22$

4.3 Detcrmining Further Correspondence and Orientation 24

5 Comments $\quad 25$

5.1 Comparison With Bium's Medial Axis Transform $\quad 25$

6 Summary and Conclusion $\quad 26$

7 Acknowledgements: $\quad 27$ 



\section{List of Figures}

Figure 1: A Rhomboidal Form and its Representation:

In the upper part of this figure the rhomboidal form is outlined in solid straight lincs. The description is for such a form which is dark on a light background. Circles indicate the locations and sizes where the band-pass filters from a sampled DOLP transform produced 3-Space peaks (M-nodes), 2-Space peaks (P-nodes), and 3-Space ridges ( L-nodes). The structurc of the resulting description is shown in the lower part of the figure. The description of the "negative shape" which surrounds this form is not presented.

Figure 2: The Resampled DOLPTransform of a Tcapot Image

Figure 3: Levels 5 Though 13 of the Resampled DOLP Transform of a Teapot Image

Figure 4: The Four Dircction Tests for Ridge-Nodes.

The four pairs of neighbors for a node in a Cartesian grid (left) and a node in a $\sqrt{2}$ grid (right) are show here. Pairs of neighbors, on opposite sides of a DOLP sample, are numbered 0 through 3 , as illustrated by the arrows. The magnitude and sign of a DOLP sample is compared to each pair of neighbors. For each direction, if neither neighbor has a DOL.P value with a larger magnitude and the same sign, then the dircction flag for that direction is set, marking the sample as a ridge-node.

Figure 5: The Direction Flags in a Band-Pass Level 7 of the Teapot Image.

This Figure shows the direction flags detected in a rcgion of band-pass level 7 of the tcapot image. Each direction flag is represented by a pair of bars poincing toward the smaller valued neighbors. Ridges tẹnd to run perpendicular to the direction flags. Peaks ( P-nodes) are marked with circles. Note that both the positive and negative peaks and ridges are shown. Note also that direction flags are not detected for nodes where the magnitude of the DOLP response is less than 5.

Figure 6: The Ridge Paths Connecting Peaks ( P-nodes ) in Band-Pass Level 7 in the Teapot Image

This figure shows the pointers connecting adjacent DOLP samples along positive and negative ridges in the crop from Band-Pass level 7 of the tea-pot image. Each pointer is represented by an arrow pointing to a neighbor node. $A$ pointer is made from a $R$-node to a neighboring $R$-node if it has a common direction flag and is a local maxima among the nearest eight neighbors. A ridge may be traced between peaks by following the pointers.

Figure 7: Positive P-Paths For Square of Size $11 \times 11$ Pixels

Figure 8: Positive P-nodes and R-paths for Level 7 of the Teapot Image 19

Figure 9: P-nodes and P-Paths for Levels 12 to 6 of the Smaller Teapot Image (teapot 1) 21

Figure 10: P-nodes and P-Paths for Levels 12 to 7 of Sccond Teapot (Scaled Larger in Size by 1.36) 23 


\section{List of Tables}

Table 1: R-Path Links for I_evels 7 and 6 of the First Teapot 22

Table 2: R-Path Links for Levels 8 and 7 of the Second Teapot (Scaled larger in Size by 1.36) 24

Table 3: Comparison of D and $\theta$ attributes for Teapots 1 and $3 \quad 24$ 


\section{Introduction}

A representation is a formal system for making explicit certain entities or types of information, and a specification of how the systen des this [20]. Representation plays a crucial role in detemining the computational complexity of an information processing problem.

This paper describes a representation for two-dimensional shape which can be used for a variety of tasks in which the shapes (or gray-level forms) in an image must be manipulated. An important property of this representation is that it makes the task of comparing the structure of two shapes to deternine the correspondence of their components computationally simple. However, this representation has uther desirable properties as well. For example, the network of symbols that describe a shape in this representation have a structure which, except for the effects of quantization, is invariant to the size, orientation, and position of a shape. Thus a shape can be compared to prototypes without having to normalize its size or orientation. An object can be tiacked in a sequence of inages by matching the largest peak(s) in its description in each image. This representation can also describe a shape when its boundaries are blurred or poorly defined or when the image has been corrupted by various sources of image noise.

This representation is based on a reversible transform referred to as the "Difference of Low-Pass" (DOL.P) Transform. From its definition, the DOLP transform of an image appcars to be very costly to compute. However several tcchniques can be used to greatly reduce the computational complexity and mernory requirement for a DOLP transform. These techniques, together with the definition of the DOIP transform. are presented in a companion paper [14].

The Difference of Low-Pass (DOLP) 'Transform is a reversible transform which converts an image intn a set of band-pass images. Each band-pass image is equivalent to a convolution of the original image with a band-pass filter, $b_{k}$. Each band-pass filter is formed by a difference of two size scaled copics of a low-pass filter, $g_{k-1}$ and $g_{k}$.

$$
b_{k}=g_{k-l}-g_{k}
$$

Each low-pass filter $g_{k}$ is a copy of the low pass filter $g_{k-1}$ scaled larger in size. These band-pass images comprise a three space (the DOLP space). The representation is constructed by detecting peaks and ridges in the DOLP space.

\subsection{Motivation:A Multi-Resolution Structural Description of Images}

Interpreting the patterns in an image requires matching. If the interpretation is restricted to twodimensional patterns, this matching is between descriptions of shapes in the inage and object models. If the interpretation is in terms of three-dimensional objects then techniques for marching among steren inages or motion sequences may be required to obtain the description of threc-dimensional shape. In cither case, the matching problem is simplified if descriptions are compared at multiple resolutions. Peaks and ridges in a DOLP Transform provide a structural description of the grey-scale shapes in an image.

The motivation for computing a structural description is to spend a fixed computational cost to transform the information in each image into a representation in which searching and matching are more efficient. In many cases the computation involved in constructing a structural description is "cgular and loca!, making the computation amenable to fast implementation in special purpose hardware. 
Several reseirchers have shown that the efficiency of cearching and matching processes can be dramatically improved by performing the search at multiple resolutions. Moravec [21] has demonstrated a multi-resolution correspondence matching algorithm for object location in sterco images. Marr and Pogrio [18] have demonstrated correspondence matching wing edges decected by a difierence of Gaussian filters at four resolutions. Rosenfeld and vanderbrug [28] have described a two stuge hierarchical template-matching algorithm. Hall has reported using a multi-resolution pyranid w dramatically speed up correlation of derial images [15]. Kelly [17], Pavlidis and Tanimoto [30], Hanson and Riscman [16], and many others liave described the use of multiple resolution images for segmentation and edge decection.

There is also experimental evidence that the visual systems of humans and other mammals separate inages into a set of "spatial frequency" channels as a first encoding of visual information. This "multi-channel theory" is based on measurements of the adaption of the threshold sensitivity to verticit sinusoidal finctions of various frequencies [10], [29]. Adaption to a sinusoid of a particular frequency affects unly the threshold sensitivity for frequencies within one octave. This evidence suggests that mammalian visial systems employ a set of band-pass channels with a band-width of about one octave. Such a set of channels would carry information from different resolutions in the image. These studies, and physiological experiments supporting the concept of parallel spatial frequency analysis, are reviewed in [9] and [31].

\subsection{Proper?ies of the Fepresentation}

The patterns which are described by this representation are "gray-scale shapes" or "forms". We prefer the term "forms". because the term shape cairics connotations of the cutline of a uniform intensity region. It is not necessary for a pattern to have a uniform intensity for is to have a well colined description in this representation. In this paper we will use the tcrn "form" to reter to the patterns in an innage.

In this representation. a form is described by a tree of symbols which represent the structure of the form at every resolution. There are four type of symbols $\{M, L, P, R\}^{1}$ which mark locations $(x, y, k)$ in the DOLP three space where a band-pass fiter of radius $R_{k}$ is a local "best-fic" to the form.

Figure 1 shows an example of the use of peaks and ridges for representing a uniform intensity form. This figure shows the outline of a dark rhomboid on a light background. Circles illustrate the position and radii of band-pass filters whose positive center lobes are a local "best-fit" to the rhomboid. Below the rhomboid is part of the graph produced by detecting and linking peaks and ridges in the sampled DOLP transform. The meaning of the symbols in this graph is described below.

A description in this representation contains a small number of symbois at the root. These symbols describe the global (or low-frequency) structure of a form. At lower levels, this tree contains increasingly larger numbers of symbols which represent more local details. The correspondence between symbols at one level in the tree constrains the possible set of correspondences at the next higher resolution level.

The description is created by detecting local positive maxima and negative minima in one dimension (ridges) and two dimensions (peaks) in each band-pass image of a DOLP transform. Local peaks in the

\footnotetext{
${ }_{1}^{1}$ previous writing about this representation, most notably in [13], these symbols were referred to by the namcs $\left\{M^{*}, L, M, P\right\}$.
} 

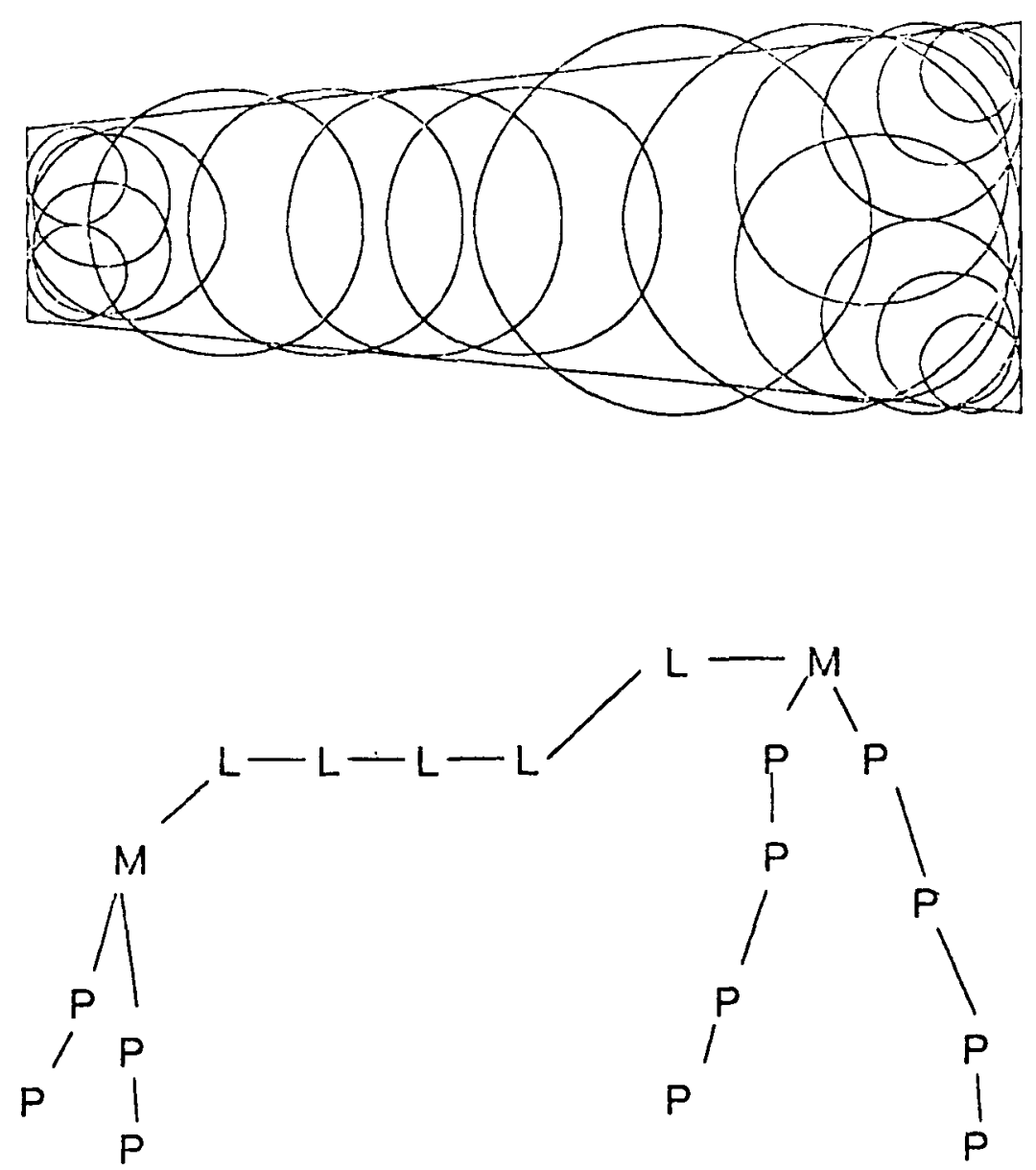

Figure 1: A Rhomboidal Form and its Representation:

In the upper part of this figure the rhomboidal form is outlined in solid straight lines. The description is for such a form which is dark on a light background. Circles indicate the locations and sizes where the band-pass filters from a sampled DOLP transform produced 3-Space peaks (M-nodes), 2-Space peaks (P-nodes), ai!d 3-Space ridges ( Lnodes). The structure of the resulting description is shown in the lower part of the figure. The description of the "negative shape" which surrounds this form is not presented.

DOLP three space define locations and sizes at which a DOLP band-pass fiiter best fits a gray scale pattern. These points are encoded as symbols which serve as landinarks for matching the information in images. Peaks of the same sign which are in adjacent positions in adjacent band-pass images are linked to furm a tree. During the linking process, the largest peak along each branch is detected. This largest peak serves as a landmark which marks the position and size of a gray-scale form. The pachs of the other peaks which are attached to such landmarks provide further description of the form, as well as continuity with structure at other resolutions. Further information is encoded by detecting and linking two-dimensional ridge points in each band-pass image and three-dimensional ridge points within the DOLP three space. The ridges in each 
band-pass image link the paiks in that image which are part of the same form. The threc-dimonsional ridges link tie largest peaks that are part of the same furn and provide a description of elongated forms.

\subsection{Correspondence iviatining}

The casiest methed for determining the cerrespondence of points in a pair of intages is to detect lanc'marks in the two images and determine une correspondence of these landmarks. The peaks and ritges in a DOL.P transform make excellent landmarks for such correspondence matching for several reasuns. These peaks and riciges provide a compact set of symbols which denote the presence and describe the shape of ferms in an image. Correspondence of symbuls of similar shapes and resolutions can be found, even is forms change shape due to motion of an object or the camera. Such peaks and ridges can also be matched when the image has been corrupled by blur or high frequency noise. Matcling can also be performed for a shape whose surface is composed of a randomi texture.

When the DOI.P transform is computed with a scalc factor of $\sqrt{2}$, there is a continuity between peaks at different levels which provides a description which varics gradually from a few symbols which describe low resolution information to the much larger number of symbols that describe high resolution details. Finding the correspondence between any pair of peaks constrains the possible correspondences of peaks under them at higher resolutions.

Segmentation techniques are used to produce symbols which represent groupings of pixels and which can act as tokens for later processing. However, the gray-scale forms that occur in an image do not. necessarily, corrcspond to individual objects, picces of objects, or surfaces in a $3-D$ scene. Furthurmore. forms witicn ate best described as a single entity at one resolution may be best described as several entities at a higher resolution. The peaks and ridges in a DOLP transform provide tokens for matching without the need for assertions about wether adjacent similar regions should be grouped together. Even if only a small set of "invariant points" of three-dimensional shapes are to be matched, the presence of these point must still be detected in the gray-scalc pattcrns of the image. Both recognition and matching of these invariaint points may be performed efficiently with peaks and ridges in the DOLP transform.

The band-pass inages in a DOI.P transform provide a multi-resulution set of symbols for representing the image gray-scale data. These symbols may be detected in each band-pass image as either the closed zerocrossing contours or the peaks and ridges within each contour. In either casc, symbols result from regions where the intensity is either darker or lighter then in surrounding regions. Each "region" will have one or more samples which are local "largest peaks" whose position in the DOLP space provides an estimate of the position and size of the region. It is not necesary for a region to be uniform to yield such peaks. Furthurmore, regions which produce a single peak at one resolution can produce more than one peak at another resolution. Finally, there is no guarantee that each peak corresponds to only one physical object, or that a particular physical object will result in a single peak.

We have observed that this representation is useful for correspondence matching to obtain thrcedimensional surface information from generalized stereo, motion, or shape from occluding contours. Stereo interpretation assumes that the gray level patterns whose shapes are compared result from the same physical three-dimensional location. This is not strictly true. Highlights on a shiny surface can move as the position of the light source or viewing angle changes. The position of shadows will change as light sources move. 
Nevertheless, correspondence matching of gray-level patterns can be a useful source of information about the shape of three-dimensional surfaces. The representation described above can simplify such correspondence matching.

\subsection{Contents of this Paper}

The following section describes the DOI.P transform. The definition of the DOL.P transform is presented, followed by a description of a fast algorithin for computing the DOLP transtorm. This fast aigerithm is bised on two independent techniques which are bricfly described. An example of a DOI.P transform of an image which contains a teapot is also provided in this section. This image will provide the data fur examples in later sections.

Section 3 describes techniques for converting the signals from a DOLP transform into a network of symbols. Processes are described for detecting points in each band-pass image which are on a ridge, or are a local peak. Techniques for linking peaks at adjacent locations in adjacent images are then described, along with a technique for detecting peaks which are local positive maxima and negative minima in the threedimensional DOLP space. A process is then described for detecting the three-dimensional ridge paths in the DOLP space.

Section 4 describes the basic principles of matching descriptions of shape by presenting a simple example in which the lower resolutiun levels of the descriptions of two teapot images are marched. The teapots in these two images differ in size by approximately 1.36 . This section illustrates the use of correspondence between the lowest resolution largest peak to decermine an estimate of the relati:c sizes and positions of the iwo objects. 'The constraint in correspondence imposed by lower resolution peaks on higher resolution peaks is then illustrated. An example of the use of the direction and length of the ridge lengthis between pcaks to determine correspundence is also presented.

\section{The Difference of Low-Pass Transform}

This section defines the Difference of Low-Pass (DOLP) transform and demonstrates its reversibility. A fast algorithm is then described for computing the DOLP transform. This fast algorithm is described in greater detail in a companion paper [14].

\subsection{The Purpose of the DOLP Transform}

The DOLP transform expresses the image information at a discrete set of resolutions in a manner which preserves all of the image information. This transform separates local forms from more global forms in a manner that makes no assumptions about the scales at which significant information occurs. The DOLP filters overlap in the frequency domain; thus there is a smooth variation from each band-pass level to the next. This "smoothness" makes size-independent matching of forms possible and makes it possible to) use the correspondence of symbols from one band-pass level to constrain the correspondence of symbols at the next ( higher resolution ) level.

The difference of two low-pass filters is a band-pass filter provided that 
1. The two filters are not identical.

2. The two filters bave both been nomalized so that their cueficients sum to 1.0 .

A filter which has a circularly symmetric pass-band that rises and then lalls monotonically will be sensitive to image information at a particular size scale. The DOLP transform cmploys a set of such filters which are exponentially scaled in size and cover the entire two-dimensional frequency spectrum.

\subsection{Definition of the DOLP transform}

The DOLP transform expands an inage signal $p(x, y)$ composed of $N=M \times M$ samples into $\operatorname{Lug}_{S}(N)$ band-pass images ${ }^{2}{ }_{B},(x, y)$. Each band-pass image is equivalent to a convolution of the image $p(x, y)$ with a band-pass impulse response $b_{k}(x, y)$.

$$
\mathscr{B}_{k}(x, y)=f(x, y) * b_{k}(x, y)
$$

For $\mathrm{k}=0$, the band-pass filter is formed by subtracting a circularly symmetric low-pass filter $g_{0}(x, y)$ from a unit sample positioned over the center coefficient at the point $(0,0)$.

$$
b_{0}(x, y)=\delta(\ddot{x}, y)-g_{0}(x, y)
$$

The filter $b_{0}(x, y)$ gives a high-pass image, $\mathfrak{B}_{0}(x, y)$. This image is equivalent to the result produced by the edge detection techinique known as "unsharp masking" [26].

$$
\begin{aligned}
\mathscr{B}_{0}(x, y) & =f(x, y) *\left(S(x, y)-g_{0}(x, y)\right) \\
& =f(x, y)-\left(p(x, y) * g_{0}(x, y)\right)
\end{aligned}
$$

For band-pass levels $1 \leq \mathrm{k}<\mathrm{K}$ the band-pass filter is formed as a difference of two size-scaled copics of the low-pass filter.

$$
b_{k}(x, y)=g_{k-l}(x, y)-g_{k}(x, y)
$$

In order for the configuration of peaks in a DOL.P transform of a form to be invariant to the size of the form, it is necessary that each low-pass filter, $g_{k}(x, y)$ be a copy of the circularly symmetric low-pass filter $g_{0}(x, y)$ scaled larger in size by a scale factor raised to the $k^{\text {th }}$ power [13]. Thus for each $k$, the band-pass impulse response, $b_{k}(x, y)$, is a size scaled copy of the band-pass impulse response, $b_{k-l}(x, y)$. For twodimensional circularly-symmetric filters which are defined by sampling a continuous function, size scaling increases the density of sample points over a fixed domain of the function. In the Gaussian filter, this increases the standard deviation, $\sigma$, relative to the image sample rate by a factor of $S_{2}^{k}$.

The scale factor is an important parameter. For a two-dimensional DOLP transform, this scale factor, denoted $S_{2}$, has a typical value of $\sqrt{2}$. It is possible to define a DOI_P transform with any scale factor $S_{2}$ for which the difference of low-pass filter provides a uscful pass band. Marr, for example, argues that a scale factor of $\mathrm{S}_{2}=1.6$ is optimum for a difference of Gaussian filters [19]. We have found that a scale factor $\mathrm{S}_{2}=$ $\sqrt{2}$ yields effectively the same band-pass filter and provides two other interesting properties [13].

${ }^{2} \mathrm{~S}$ is the square of the scale factor 
First, resmpling each bund-pass inage at a sample distance which is a fixed fraction of the filcer's size provides a configuration of peiks and ridges in each band-pass imatge which is invariant whe size of the object, exespt for the effects of quantization. Thus the resample distance and the scate factor should to the same value. The smallest distance at which a two-dinensional signal can be resanpled is $\sqrt{2}$. Sccond. a DOL.P transtorm can be computed using Gaussian low-pass filters. The convolution of a Gaussian filter with itself produces a new Gaussian filter which is scaled larger in size by a factor of $\sqrt{2}$. These (wo properties make $\sqrt{2}$ a convenient valuc for both the scale factor and the resample distance.

In principle the DOLP tranitorm can be defined for any number of band-pass levels $K$. $A$ convenient value of $K$ is

$$
\mathrm{K}=\log _{\mathrm{S}}(\mathrm{N})
$$

Where the value $S$ is the square of the sample distance $S_{2}$.

$$
\mathrm{S}=\mathrm{S}_{2}^{2}
$$

This value of $K$ is the number of band-pass images that result if cach band-pass image, ${ }^{6} \mathscr{B}_{\mathrm{k}}$, is resampled at a sampling distance of $\mathrm{S}_{2}^{\mathrm{k}}$. With this resampling, the $\mathrm{K}^{\text {th }}$ image contains only one sample.

The DOL.P transform is reversible which proves that no information is lost. The original inage may be recovcred by adting all of the band-pass images, plus a low-pass residue. This low pass residue, which has not been found to be uscfiul for describing the inage, is the convolution of the lowest frequency (largest) low-pass filter. $g_{K}(x, y)$ with the image.

$$
p(x, y)=\left(p(x, y) * g_{K}(x, y)\right)+\sum_{k=0}^{\mathbf{K}-1} \mathfrak{B}_{k}(x, y)
$$

\subsection{Fast Computation Techniques: Resampling and Cascade Convolution}

A full DOLP transform of an image composed of $N$ samples, produces $K=\log _{S}(N)$ band-pass images of $\mathrm{N}$ samples each, and requircs $\mathrm{O}\left(\mathrm{N}^{2}\right)$ multiplics and additions. Two techniques can be used to reduce the computational complexity of the DOLP transform: "resampling" and "cascaded convolution with expansion".

Resampling is based on the fact that the filters used in a DOLP transform are scaled copies of a bandlimited filter. As the fitter's impulse response becomes larger, its upper cutoff frequency decreases, and thus its output can be resampled with coarser spacing without loss of information. The exponential growth in the number of filter coefficients which results from the exponential scaling of size is offset by an exponential growth in distance between points at which the convolution is computed. The result is that cach band-pass image may be computed with the same number of multiplications and additions. Resampling each band pass image at a distance of $\sqrt{2}$ reduces the total number of points in the DOLP space from $N \log _{S}(N)$ samples to $3 \mathrm{~N}$ samples.

Cascaded convolution exploits the fact that the convolution of a Gaussian function with itself produces a Gaussian scalcd larger by $\sqrt{2}$. This method also cmploys "expansion", in which the coefficients of a filter are 
mapped into a larger sample grid, thercby expanding the size of the filter, at the cost of introducing reflections of the pass region about a new Nyquist boundary in the transfer function of the filter. This operation docs not introduce distortion, provided the filter is designed so that the reflections of the pass region fall on the stop region of the composite filter and are sufficiencly attentiated so as to have a negligible cffect on the composite filter. Thus a scquence of low-pass images are formed by repeatedly convolving the image with each expanded version of the low-pass filter $g_{0}$. Each expansion of the low-pass filter maps its coefficients onto a sample grid with a spacing between samples increased $\sqrt{2}$. Thus each low-pass image has an impuise response which is $\sqrt{2}$ larger than that of the previous image in the sequence. Each low-pass image is then subtracted from the previous low-pass image to form the band-pass images.

Combining these two techniques gives an algorithm which will compute a DOLP transform of an $\mathrm{N}$ sample signal in $\mathrm{O}(\mathrm{N})$ multiplies, producing $3 \mathrm{~N}$ sample points. This algorithm is described in [14]. In this algorithm, each low-pass image is resampled at $\sqrt{2}$ and then convolved with the low-pass filter $g_{0}$ to form the next low-pass intage. Since each low-pass image has half the number of samples as the previous lo'v-pass image, and the number of filter coefficients is constant, each low-pass image is computed from the previous low-pass image using half the number of multiplies and additions. Thus, if $\mathrm{C}_{0}$ is the number of multiplies required to compute low-pass image 0 , the total number of multiplies needed to compute $\mathrm{K}$ band-pass levels is given by:

$$
\begin{aligned}
\mathrm{C}_{\text {Tot }} & =\mathrm{C}_{0}(1+1+1 / 2+1 / 4+1 / 8+1 / 16+\ldots+1 / \mathrm{K}) \\
& =3 \mathrm{C}_{0}
\end{aligned}
$$

Each low-pass image is then subtracted from the resampled version of the previous low-pass inage to form the band-pass inage. Thus each band-pass image has a sample density which is proportional to the size of its impulse response.

\subsection{An Example: the DOLP Transform of a Teapot Image}

Figure 2 shows a DOLP transform of an image of a teapot that was produced using the fast compucation techniques described above. In this figure the image at the lower right is the high frequency image, $\mathfrak{B}_{\mathrm{o}}(x, y)$. The upper left corner shows the level 1 band-pass image, $\mathscr{B}_{1}(x, y)$, while the upper right hand corner contains the level 2 band-pass image, $\mathscr{B}_{2}(x, y)$. Underneath the level 1 band pass image are levels 3 and 4 , then 5 and 6 , etc. Figure 3 shows an enlarged view of band-pass levels 5 through 13. This enlargement illustrates the unique peaks in the low frequency images that occur for each gray-scale form.

The use of $\sqrt{2}$ resampling is apparent from the reduction in size for each image from level 3 to 13 . Each even numbered image is actually on a $\sqrt{2}$ sample grid. To display these $\sqrt{2}$ images, each pixcl is printed twice, creating the interlocking brick texture evident in Figure 3.

\section{Construction of the Representation from a DOLP Transform}

In this section we describe techniques for constructing the representation for grav-scale forms. This construction process is described as a sequence of steps in which peaks and ridges are first detected arid linked in each band-pass image, and the resulting symbols are then linked among the band pass levels. 


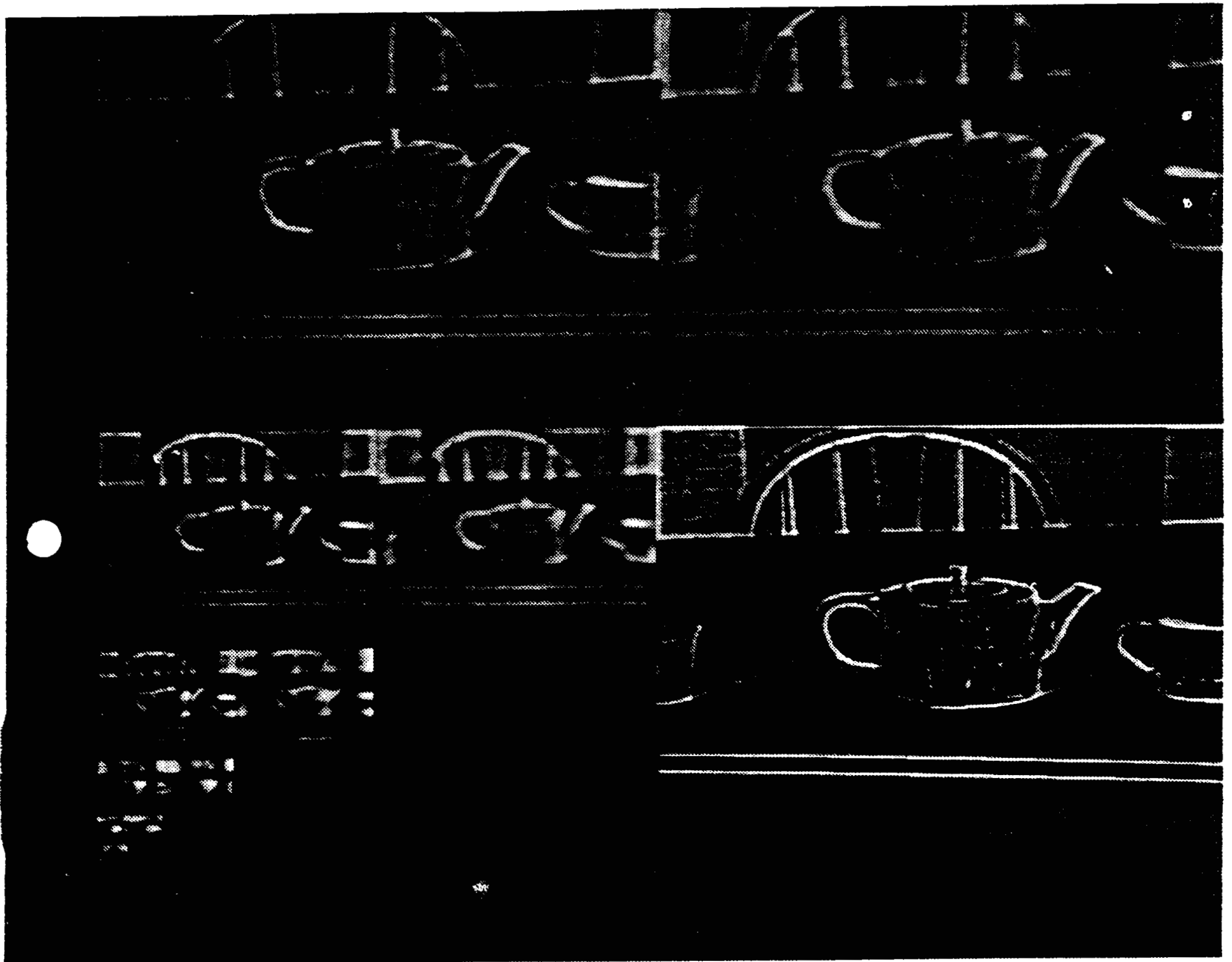

Figure 2: The Resampled DOLP 'Transform of a Teapot Image 


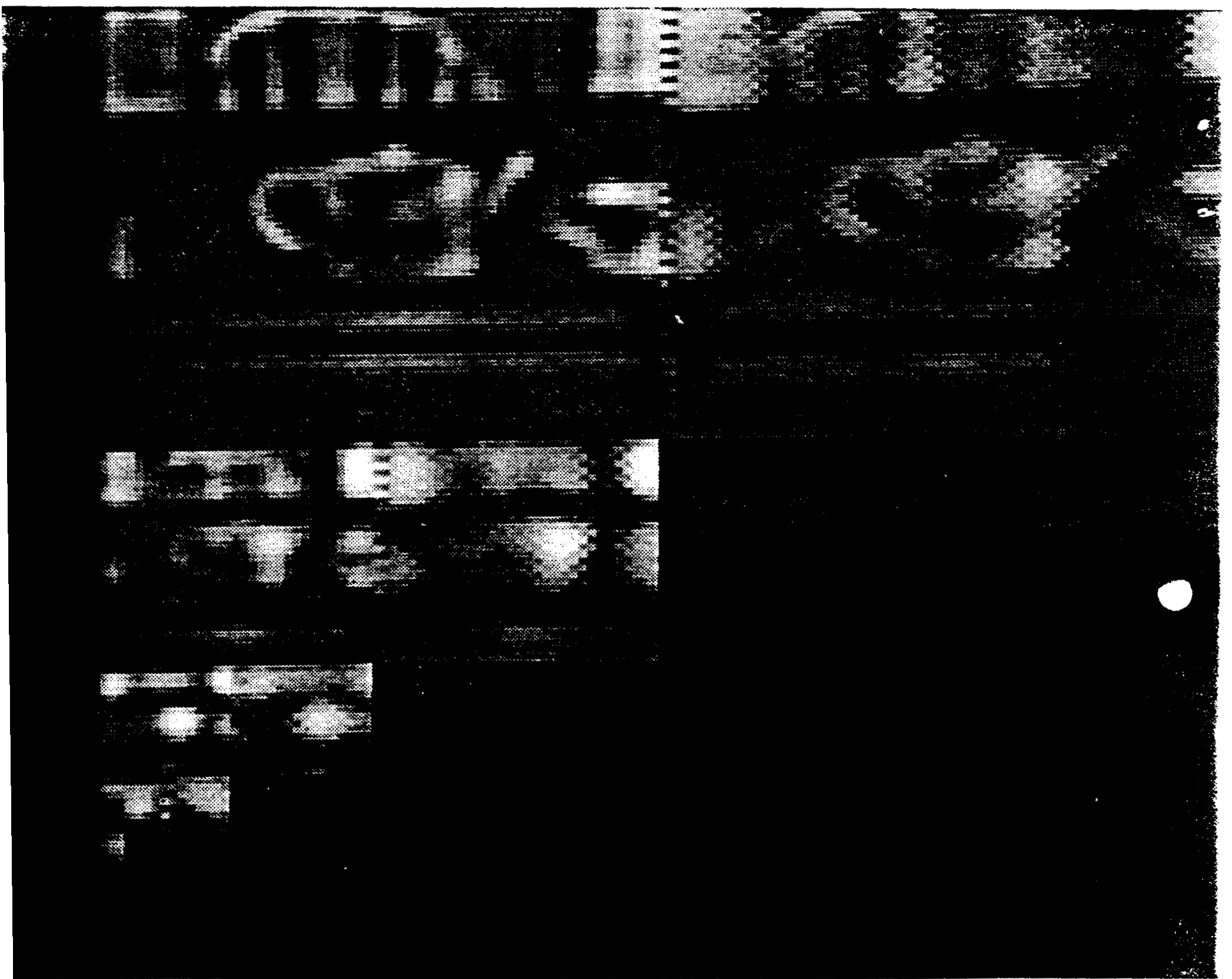

Figure 3: Levels 5 Though 13 of the Resampled DOLP'lransform of a T'apot Image 


\subsubsection{The Approach}

Peaks and ridges mark locations where the DOLP inpulse responses are a "best fit" to the image data. This "best-fit" paradigm is based on the obscrvation that, for a circularly symmetric filter. corculation and convolution are equivalent operations. Furthemore, a correlation is compused of a sequence of inner preducts besween the filter coefficients and neighborhoods (of the same size as the filter support) in the image. Thus peaks in the convolution are locations where the impulse response correlates (is a local best fit) to the image. Ridges are a sequence of locations where the filters are a "gond fit" to the innge ditta. We may think of the DOI.P band-pass impulse responses as a set of "primitive" functions for representing forms in an image.

The "local neighborhood" of a DOL.P sample is the nearest eight neighbors on the simple grid at its band-pass level. A "peak" (or P-node) is a local positive maxima or negative minima within a twodimensional band-pass image. A "ridge-node" (or R-node) is a local one-dimensicnal positive maximum or negative mininlum within a two-dimensional band-pass image. Peaks within a form are linked by paths of largest ridge-nodes ( R-patis ).

In order for a DOLP sample to be a local positive maximun or negative minimum in the DOLP threespace, it must also ce a local peak within its band-pass level. Furthermore, for a sample to be a peak in its band-pass level, it must be a ridge-nude in the four directions given by opposite pairs of its cight neighbors. Peaks and ridge-nodes are first detected within each band-pass image. Peaks are then linked to peaks at adjacent levels to furm a tuce of symibols (composed of a paths of peaks, or P-paths). During this linking it is possible to detect the peaks which are local positive maxima and negative minima in the DOLP three-space. The three-space poaks are referred to as M-noules.

The ridge-nodes are also linked to form rilge-paths in each band-pass inage (called R-paths) and in the DOI.P three-space (called I,-paths). The ridges in the DOI P thres-space ( L-paths) describe elongated forms and connect the largest peaks ( M-nodes) which are part of the same form.

The process for constructing a description is composed of the following stages:

1. Detect ridge-nodes (R-nodes) and peaks (P-nodes) at each band-pass level;

2. Link the largest adjacent ridge-nodes with the same direction flags in a band-pass level to form ridges ( R-paths) which connect the P-nudes in that level;

3. Link two-dimensional peaks ( P-nodes) at adjacent positions in adjacent levels to form P-paths;

4. Detect local inaxima along each P-path (M-nodes);

5. Detect the ridge nodes ( $R-n o d e s)$ which have larger DOLP values than those at neighboring locations in adjacent images to detect I-nodes.

6. Link the largest adjacent ridge points with the same direction among the band-pass levels to form three-dimensional ridge paths (L-paths). 
The result of this proces is a tree-like graph which concains four claces of symbols:

- R-nodes: DOLP Sumples which are on a ridge at a level.

- P-nodes: DOLP Sanples which are local two-dimensional maxima at a level.

- L-nodes: DOLP samples which are on a ridge across levels (i.e. in the three space $(x, y, k)$ ).

- M-nodes: Points which are local maxima in the three space.

Evcry uniform (or approximately uniform) region will have one or. more M-nodes as a root in its description. These are connected to paths of L's (L-Paths) which describe the gencra! form of the region, and paths of P-nodes (P-Pachs) which branch into the concavitics and convexitics. L-paths terminate at other M-nodes which describe significant features at higher resolutions. The shape of the boundaries are described in multiple resulutions by the ridges at each band-pass level ( $R$-paths). If a boundary is blurry, then the highest resolution (iowest-level) $R$-paths are lost, but the boundary is still described by the lower resolution R-paths.

\subsection{Deiection of Peak-Nodes and Ridge-Nodes within each Band-pass Image}

Peak-nodes and ridge-nodes in each band-pass level are detected by cumparing the magnitude and sign of each sample with the magnitude and sign of opposite pairs of its eight nearest ncighisors. This comparison is made in four directions, as indicated hy Figure 4, aid can result in one of four "dicecion flags" being set. A direstion flag is set when neither ncighbor sample in a direction has a DOLP value of the siune sign and a larger magnitude.

If any of the four direction flugs are set, then the sample is encuded as a R-node. If all four direction flags have been set then the sample is encoded as an P-node. The direction llags are saved to be used to guide the processes for detecting two-dimensional ridges ( $R$-paths) and three-dimensional ridges (L-paths).

Two possibilities complicate this rather simple process. When the amplitude of the signal is very small, it is possible to have a small region of adjacent samples with the same DOLP sample value. Such a plateau region may be avoided by not setting direction flags for samples with a magnitude less then a small thieshold. $A$ value 5 has been found to work well for 8 bit DOLP samples. Also, it is possible to have two adjacent samples with equal DOI.P values, while only one has a neighbor with a larger magnitude. Such cases may be easily detected and corrected by a local two stage process. The correction involves turning off the direction flag for the neiginbor without a larger neighbor.

Figure 5 shows the direction flags detected in a region from band-pass level 7 of the Teapor image. Each direction flag which is set is represented as a pair of short line segments on both sides of a sample. These line segments point in the direction in which the sample is a one-dimensional maxima. Samples which are two-dimensional peaks ( P-nodes) are marked with a circle. It is possible to implement this detection in parallel or with a fast serial procedure. 


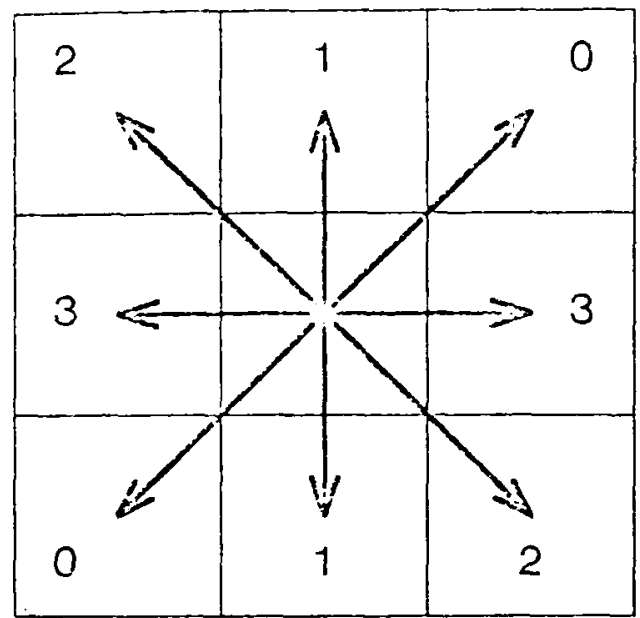

Cartesian Grid

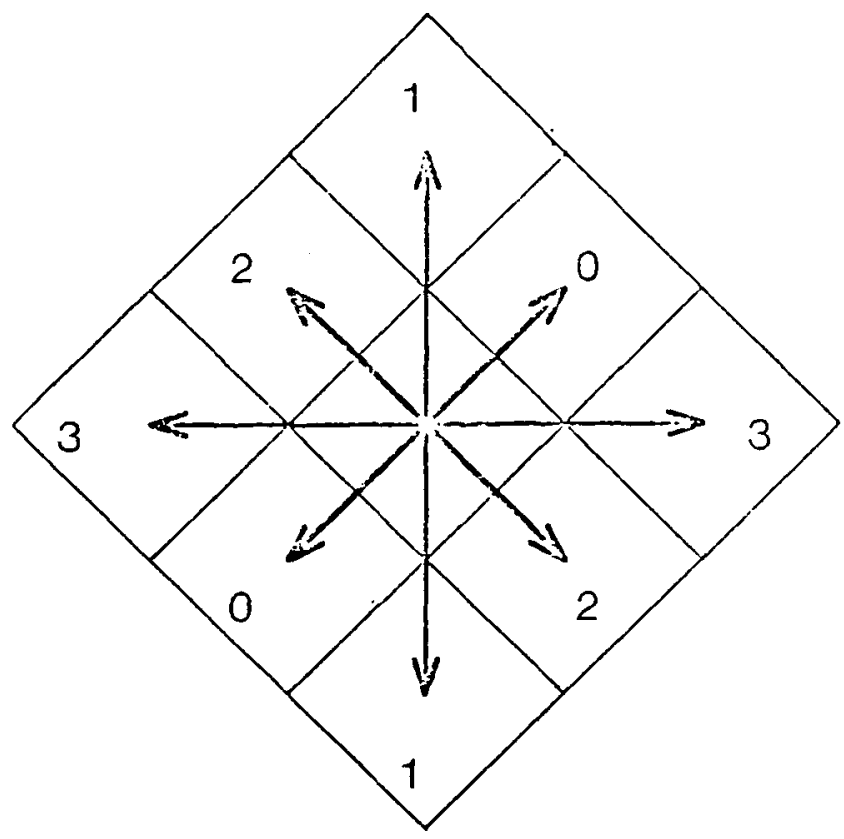

Square Root(2) Grid

Figure 4:

The Four Direction Tesis for Ridge-Nodes.

The four pairs of neighbors for a node in a Cartesian grid (left) and a node in a $\sqrt{2}$ grid (right) are show here. Pairs of neighbors, cn upposite sides of at DOLP sariple, are numbered 0 through 3 , as illustratcd by the arrows. The magnitude and sign of a DOL.P sample is compared to each pair of neighbors. For each direction, if neither neighbor has a DOLP value with a larger magnitude and the same sign, then the direction flag for that direction is set, marking the sample as a ridge-node. .

\subsection{Linking of Ridge-Paths at a Band-Pass Level}

There are two purposes for which ridge paths in a two-dimensional band-pass level are detected:

1. To provide a link between P-nodes at a level which are part of the same form, and,

2. to construct a description of the boundary of a form.

I.inking P-nodes of the same sign and band-pass level with ridges provides information about the connectivity of a form and provides attributes of distance and relative orientation which can be used in determining correspondences of P-nodes across levels.

In general, when a boundary is not a straight line, the convexities and concavities are described by a P-path. 


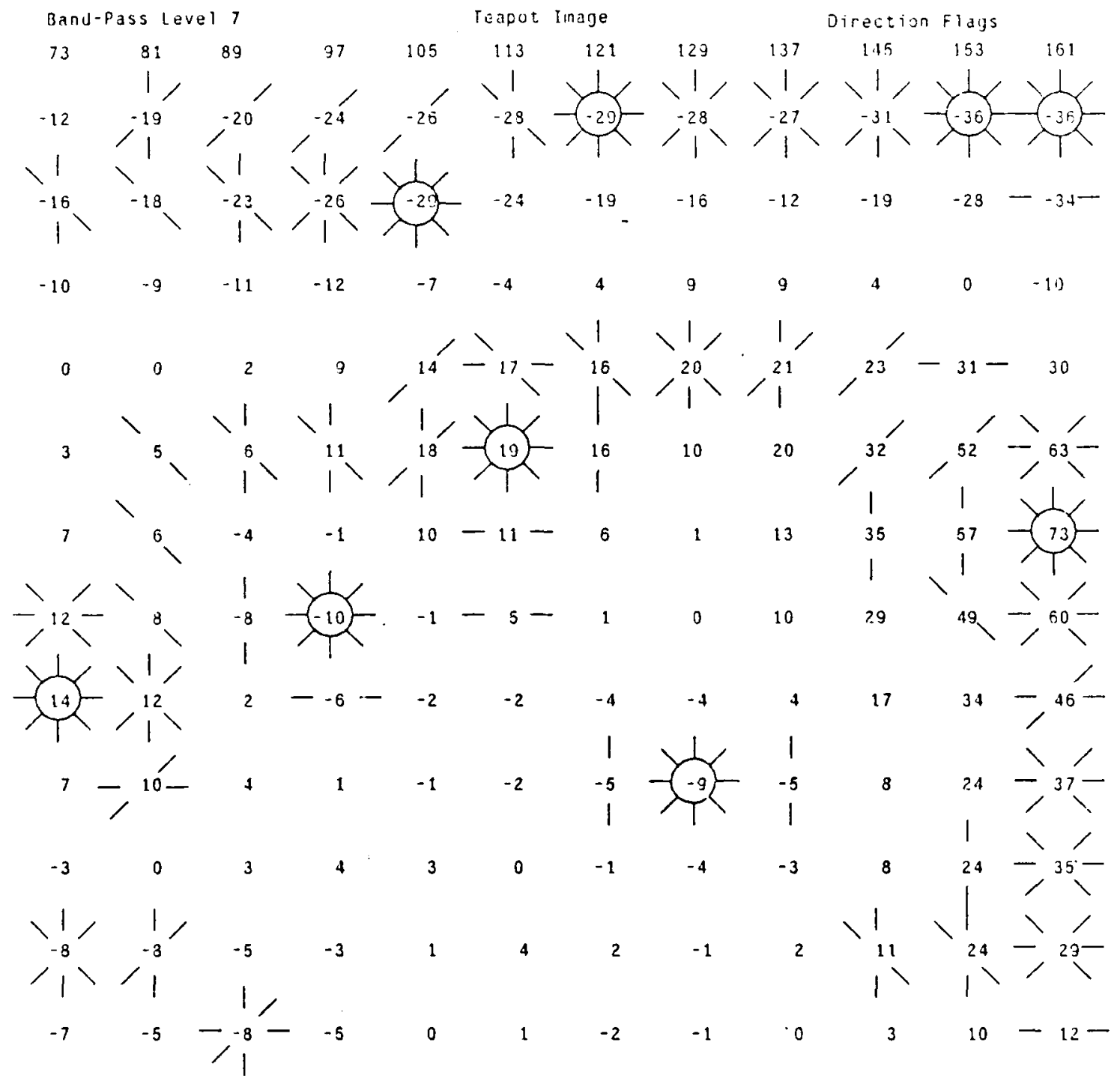

Figure 5:

The Direction Flags in a Band-Pass Level 7 of the Teapot Image.

This Figure shows the direction flags cetected in a region of band-pass level 7 of the teapot image. Each direction flag is represented by a pair of bars pointing toward the smaller valued neighbors. Ridges tend to run perpendicular to the direction flags. Peaks ( P-nodes) are marked with circles. Note that both the positive and negative pcaks and ridges are shown. Note also that dircetion flags are not detected for nodes where the magnitude of the DOLP response is less than 5. 


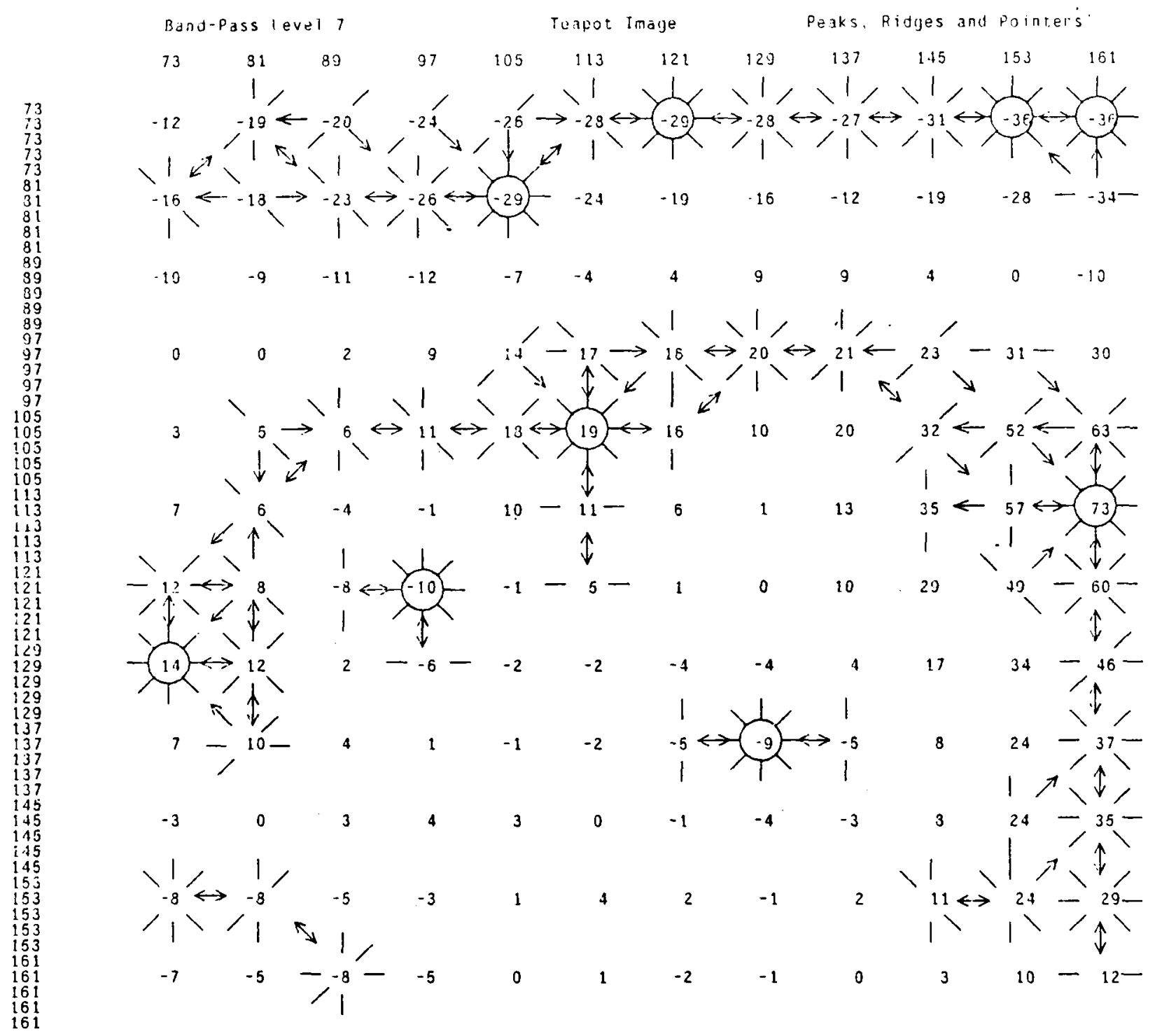

Figure 6:

The Ridge Paths Connecting Fraks ( P-nodes) in Band-Pass

Level 7 in the Teapot Image

This figure shows the pointers connecting adjacent DOLP samples along positive and negative ridges in the crop from Band-Pass level 7 of the tea-pot image. Each pointer is represented by an arrow pointing to a neighbor node. $A$ pointer is made from a $R$-node to a neighboring $\mathrm{R}$-node if it has a common direction flag and is a local maxima among the nearest eight neighbors. A ridge may be traced between peaks by following the pointers. 
However, when the curvalure is very gradual P-nodes may no occur for the concavities and combexities. In either case, a precise description of the location of the boundary is provided at multiple resolutions by the path of the ridge in a band-pass level.

$A$ ridge is the path of largest $R$-nodes between $P$-nodes. This path can be formed by a local linking process which is executed independently it each $R$-node. The ridje path can be detected by having each $R$-node make a pointer to neighboring $R$-nedes which mect two conditions:

1. The neighbor R-node has the same sign and direction flags; and,

2. The magnitude of the DOI.P sample at the neighboring $R$-nude is a local maximum in a linear list of DOI P values of neighbors.

An earlier, more complex algorithm for the same purpose was described in [13]. The result of this process when applied to the level 7 band-pass image is shown in Figure 6.

\subsection{Linking Peaks Belween Levels and Detecting the Largest Peak}

The band-pass filters which compose a DOLP transform are denscily packed in the frequency domain. Each filter has a signilicant overlap in the pass-band of its transfer function with the band-pass filters from neighboring levels. As a result when a form results in a two-dimensional peak (or P-node) at one band-pass levil the iilters at adjacent levels will tend to causc a peak of the sane sign to occur at the sanse or adjacent positioris. Connecting P-nodes of the same sign which are at adjacent locations in adjacent band-pass inages yields a sequence of P-nodes referred to as a P-path. P-Paths tend to converge at lower resolutions, which gives the description the form of a tree. The branches at higher resolution of this trec describe the form of "rourdish" biobs, bar-ends. corners and pointed protrusions, and the patterns of concavities and convexities along a boundary. Descending. Lie tree of P-paths in a description gives an increasingly more complex and hisher resolution description of the form.

The magnitude of the DOLP filter response of P-nodes along a P-path tend to rise monotonically to a largest magnitude, and then drop off monotonically. This largest value is encoded as an M-node. Such nodes serve as landmarks for matching descriptions. An M-node gives an estimate of the size and position of a form or a significant component of a form. Determining the correspondence of parts of forms in two descriptions is primarily a protlem of finding the correspondence between M-nodes and the L-paths which connect them.

A simple rechnique may be used to simultaneously link P-nodes into a P-path and detect the $\mathrm{M}$-nude (largest P-node) along each P-path. This technique is applicd iteratively for each level, starting at the next to the lowest resolution level of the DOLP transform (level K-2). The technique can be implemented in parallel within each level. This technique works as follows. Starting at eacn P-node at level $\mathrm{k}$, the nearcst upper ncighbors at level $k+1$ are examined to sec if they are also $P$-nodes of the same sign. If so, a two-way pointer is made between these two P-nodes.

It is possible for P-nodes that describe the same form at two adjacent levels to be separated by as much as 
two samples. Thus, if no P-ncdes are found in the nearest 4 or 8 ncighburs it level $k+1$ for a P-node at level $k$. dhen the nodes in the larger neighborhood given by the neighbors of the neighbors is examined. $A$ iwo-way pointer is made for any P-nodes found in this larser neighborhood.

During this linking process it is also possible to detect the largest P-nodes on a P-path by a process referred to as "flag-stealing". This technique requires that P-node linking oecur serially by level. In the fliag stealing process, a P-node with no upper neighbor or with a magnitude greater or equal to all of its upper neighbors sets a flag which indicates that it is an M-node. Peaks which are adjacent to it at lower levels cun "steal" this flag if they have an rqual or larzer maynitude. When the flag is stolen, the lower node sets its own flag as well as setting a second flig in the upper P-node which is then used to eancel the flag. This two stige process permits the W-flag to propigate down multiple branches if the P-path splits.

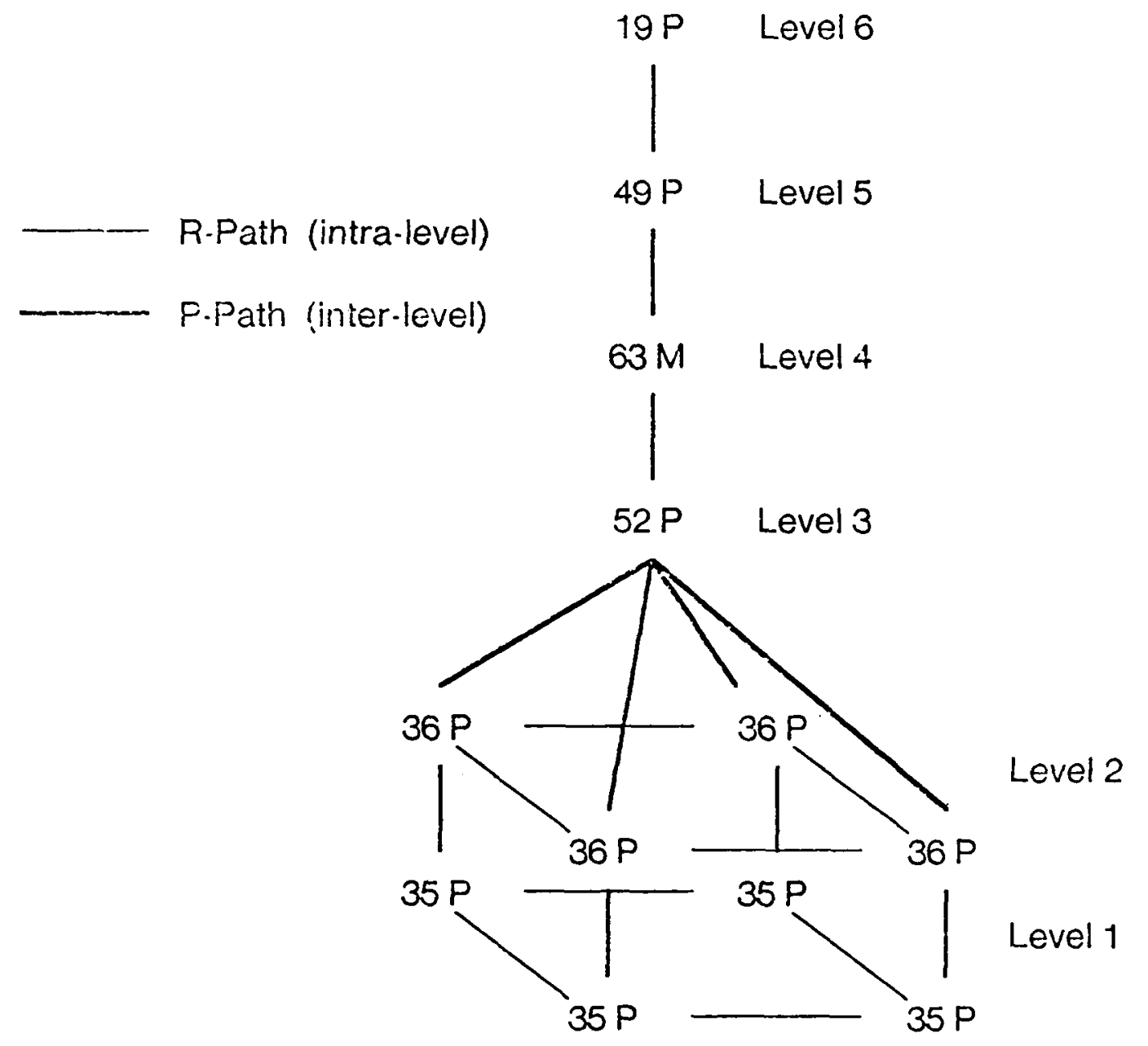

Figure 7: Positive P-Paths For Squarc of Sizc $11 \times 11$ Pixcls

Figure 7 shows the P-paths and the M-node that occur at level 6 through 1 for a uniform intensity square of $11 \times 11$ pixcls, and grey level 96 on a background of 32 . The reader can simulate the P-node linking and flag stcaling process with this figure. The process starts at level 6, where the P-node has a valtie of 19 .

${ }^{3}$ The two possible upper neighborhoods in the DOLP space with $\sqrt{2}$ sampling. 


\subsection{Detecting the Largest Three-Dimensional Ridge Path}

Three-dimensional ridges are essential for describing forms which are clongated. An elongated form almost always has an M-nede at each end. and a ridge of large DOL.P valus connecting the two W-nodes. The DOL.P values along this ridge end to be larger than than those along the ridges in the band-pass levels above and below, because the pusitive center coefficients of the band-pass for that level "fit" the wide of the clongated form. Where the form grows wider, the largest ridge will move to a higher (coarser) band-pass level. Where the form grows thinner, the largest ridge will move to a lower (smaller resolution) band-pass level. This ridge of largest DOI.P samples is called an I.-path and the norles along it are called I-nodes. $\mathrm{L}$-nodes are $\mathrm{R}$-nodes that are larger than their neighbors at adjacent band-pass levels.

L-nodes may be detected by a process similar to the flag-stcaling process used to detect the largest peak, or M-node along a $P$-path. That is, starting at the band-pass level below the lowest resolution, each $R$-node cxamines a neighborhood in the level above it. An R-node is determined to be an L-node if is has a larger value than the $R$-nodes in approximately the same place in the ridges above and below it.

Thus each R-node scans an area of the band-pass level above it. This area is above and to the sides of its ridge. The magnitudes of DOLP samples of the same sign found in the neighborhood in the upper ridge are compared to that of the R-mode, and a flag is set in the lower R-node and cleared in the upper R-node if the lower $R$-node is smaller. In this way, the $L$ - - hags propagate down to the level with the larest DOL $P$ sainples along the ridge. I,-nodes are linked to form I-paths, by having each L-node sean its three-dimensional neighborhood and link to L-nodes which have the same sign and are local maxima in the thrce-dimensional DOLP space neighoorhood.

\section{A Simple Example of Matching}

There are many applications for shape matching, and each application demands matching algorithms with certain properties. This section does not provide a matching algorithm. Instcad, it describes some principles about matching forms that have been encoded in the representation described above. Primarily, these principles involve techniques for discovering the correspondence between "landmark" symbols in the two descriptions. A fundamental principle is that the correspondence of $\mathrm{P}$-nodes and $\mathrm{M}$-nodes in two descriptions is constrained by the correspondence of $\mathrm{P}$-nodes and $\mathrm{M}$-nodes at coarser resolutions in the same $\mathrm{P}$-path.

As an example of correspondence matching using this representation, this section shows the process of discovering the correspondence between the coarsest resolution P-nodes in two images of a teapot taken with a change in distance betwcen the teapot and the camera by a factor of 1.36. In this example matching is shown for the P-nodes from the most global level (level 12) to the second highest level with more than one P-node.

The first image is referred to as teapot image 1 . This is the image whose sampled DOL.P transform is shown in the examples in figures 2 and 3. The P-nodes for levels 12 through 6 of teapot image 1 were hand matched to those of the second teapot image, referred to below as teapot 2. Other examples of M-node matching for the teapot images are given in [13]. 


\subsection{Abstracting the Graph cf Connected Peaks at a Level}

The algorithms described above are all presented from the point of view of hasing data which is "cmledded" in the DOLP space. To obcain a description of gray-scale shape which is general purpose it is desirable to construct a graph which not embedded in the DOL.P space. Such a descriptiun may be stored with much less memory.

The primary skcleton of such a description is the tree of P-paths and the interconnecting L-paths. The $P$-nodes at each band-pass kevel are linked to other P-nodes of the same sign and level which are part of the same form. This linking is provided by tracing the R-paths that connect P-nodes at a level. Each link is cncoded as a two-way pointer between P-nodes.

Each P-node and $M$-node has attribules of its DOLP sample value and its position $(x, y, k)$ in the DOLP space. Connected P-nedes are "linked" by two way pointers. Each half of a pointer may also be assigned the attributes of distance (D) and orientation $(\partial)$, which are defined as:

Distance: The distance between two P-nodes is the cartesian distance measured in terms of the number of samples at that level. In levels with a $\sqrt{2}$ sample grid, the distance along the $x$ and $y$ axes are in units of $\sqrt{2}$.

Orientation: The orientation between two P-nodes is the angle between the line that conricets diem and the $x$ axis in the positive direction.

The attributes of distance and orientation are useful for determining the correspondence betwecin smali groups of P-nodes from t:vo DOLP transforms.

\subsubsection{Example of Abstracted P-nodes and R-paths}

The P-nodes and R-nodes from level 7 of the teapot image are shown above in Figure 6. Level 7 is the highest level with more than one P-node describing the tcapot.

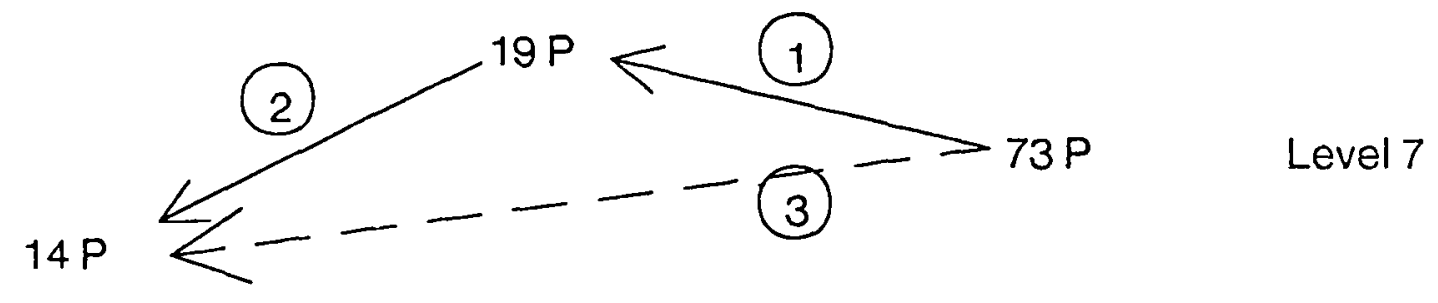

Figure 8: Positive P-nodes and R-paths for Level 7 of the Tenpot Image

The three positive peaks from level 7 of the teapot image are shown abstracted from the band-pass data in Figure 8. The R-path links between these P-nodes are illustrated with arrows and labeled with circled numbers, called "Link numbers". Links 1 and 2 are examples of "directly" connected P-nodes. A pair of 
P-nodes are directly connected when they are connected by an R-path with no intervening P-nodes between then. The R-path link between the right-most and left-most P-nudes is shown as a dotted arrow labeled as link 3. Link 3 shows an example of a pair of "indirecty" connected P-nodes. Including indirect R-path links in matching P-nodes prevents the matching algorithm from errors caused by missing or cxtrancous $P$-nodes.

In this early matching experiment, special status was given to the P-nodes along the "principal P-path". This is the P-path which includes the highest $M$-node. Thus arrows and indirect links are shown cmanating from the P-node from this P-path. In our more recent experiments, all links are two-way, and indirect links are made for all P-nodes which are not at the top of a P-path.

The link numbers are also used as an index into a table of attributes. The attributes fer these particular links are given in table 1 in the next section. This same set of links is included in Figure 9. These numbers are also used to show the correspondence which was assigned by hand matching between these links and the same links in the larger teapot image.

These attribute tables give the values for $\mathrm{dx}, \mathrm{dy}, \mathrm{D}$, and $\theta$ for each R-path link. The positive directions for $\mathrm{dx}$ and $\mathrm{dy}$ are the same as used in the image: $+\mathrm{x}$ points right, $+\mathrm{y}$ points down. Note that $\theta$ increases in the counter-clockwise direction. In these tables, in the levels which are at a $\sqrt{2}$ sample grid, the distances $d x$ and dy are recorded in units of $\sqrt{2}$. In cases where a P-node spans two adjacent samples, the P-nodes position is assigned at the mid-point beiwein them. This results in values of $\mathrm{dx}$ or dy that have fractional parts of 5 in the cartesian-sampled (odd) levels, and $.25, .5$ or .75 in the $\sqrt{2}$-sampled (cven) levels.

In tables 1 and 2 , orientation $(\theta)$ is measured in degrees. On a cartesian grid. at distances that are typically 5 to 10 pixcls, angular resolution is typically 5 to 10 degrees. Of course, the longer the distance, the more accurate the estimate of orientation.

The P-nudes for levels 12 through 6 of the teapot image are shown in Figure 9. In levels 12 through 9 of Figure 9 only a single P-node occurs in the teapot. These P-nodes all occur within a distance of two samples of the P-node above thein, and are thus linked into a single P-Path. ${ }^{4}$ This P-path is referred to as the principal P-Path. The P-nude at level 8 has the largest value along this P-path and is thus marked as an $\mathrm{M}$-node. This P-node corresponds to a filter with a positive center lobe of radius $R_{+} \approx 18$ pixels or a diameter of 37 pixels. This corresponds to the form in the image that results from the ovcrlap of the shadow on the right side of the teapot and the darkly glazed upper half of the teapot. ${ }^{5}$ At level 7, additional detail begins to emerge. P-nodes occur over the upper right corner of the teapot and over the handle region. These P-nodes are joined to the P-node on the principal P-path by an R-Path.

Five P-nodes occur in level 6. Three of these P-nodes occur underneath (within 2 samples of) P-nodes from level 7. These three P-nodes are thus part of three P-paths. The remaining two P-nodes are in fact the highest levels of two more P-paths. The P-path that begins at level 12 is referred to as the principal P-path. Only the indirect links between the principal $P$-path and a subset of the other P-nodes are shown in this figure and used in the matching example.

\footnotetext{
${ }^{4}$ The P-path links appear as vertical dark lines in figure 9 although in fact there can be a lateral shift of up to two samples between their positions.

${ }^{5}$ The tcapot images were digitized from negatives. Thus dark forms appear light in Figures 2 and 3.
} 
R.Paih (intra-level)

P.Path (inter-level)

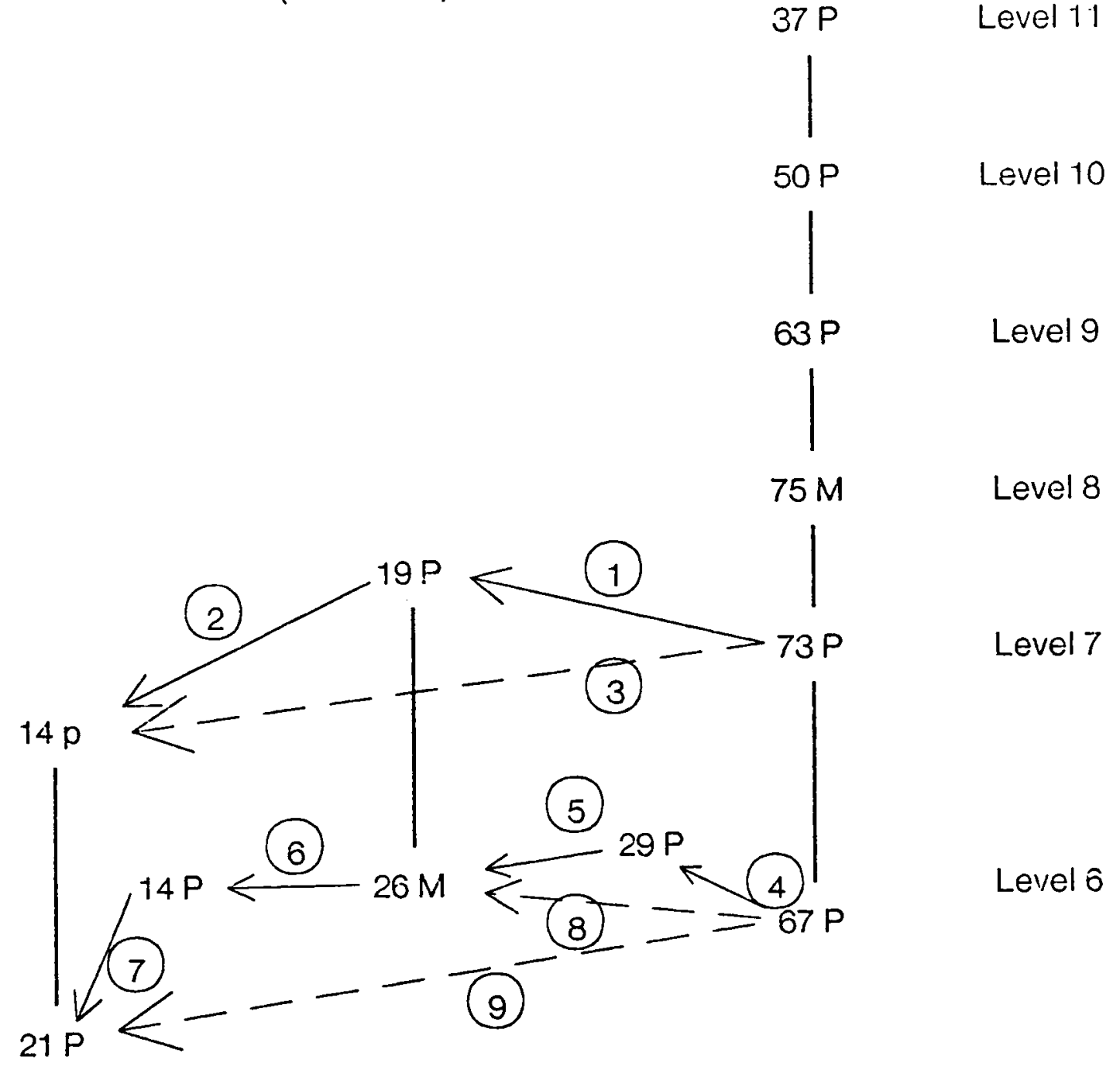

Figure 9: P-nodes and P-Paths for Levels 12 to 6 of the Smaller Teapot Image (teapot 1) 


\begin{tabular}{llllll} 
R-Path & I.crel & $\mathrm{dx}$ & $\mathrm{dy}$ & $\mathrm{I}$ & $\theta$ \\
\hline 1 & 7 & -6 & -2 & 6.32 & $161.5^{\circ}$ \\
2 & 7 & -5 & 3 & 5.83 & $210.9^{\circ}$ \\
$3(1 \& 2)$ & 7 & -11 & 1 & 11.04 & $185.2^{\circ}$ \\
4 & 6 & $-4.0 \sqrt{2}$ & $-2.0 \sqrt{2}$ & 6.32 & $153.4^{\circ}$ \\
5 & 6 & $-3.25 \sqrt{2}$ & $1.5 \sqrt{2}$ & 5.06 & $205.8^{\circ}$ \\
6 & 6 & $-3.0 \sqrt{2}$ & 0.0 & 4.24 & $180^{\circ}$ \\
7 & 6 & $0.25 \sqrt{2}$ & $3.25 \sqrt{2}$ & 4.6 & $265.6^{\circ}$ \\
$8(4 \& 5)$ & 6 & $-7.25 \sqrt{2}$ & $-0.5 \sqrt{2}$ & 10.2 & $176.1^{\circ}$ \\
$9(4 \& 5 \& 6 \& 7)$ & 6 & $-10 \sqrt{2}$ & $2.75 \sqrt{2}$ & 14.6 & $195.3^{\circ}$
\end{tabular}

Table 1: R-Path Links for Levels 7 and 6 of the First Teapot

Note that an M-node occurs at level 6 . This M-node corresponds to the upper left corner of the teapot and marks the left end of the dark region of glaze on the upper half of the teapot. The width of the positive center lobe of the filter which corresponds to this $\mathrm{M}$-node gives an approximation of the width of the darkly glazed icgion.

\section{$\therefore 2$ Initial Aligriaient is Obtain Size and Position}

In matching two forms it is convenient to designate one form as a "reference form" and the other as a "data form". One then speaks of rutating, translating and scaling the reference form so that its elements are brought into correspondence with the data form. In the examples presented below, teapot 1 is considered as the reference form which is transforned to match the teapot 2 (the data form).

Initial estimates of the alignment and relative sizes of two gray scale forns may be constructed by making a correspondence betreen their highest level P-nodes. This is illustrated by comparing the P-nodes and links in Figure 9 to those in Figure 10 shown below. Figure 10 shows the P-nodes and P-Path links for a tcaput from a second image. This size scaling was accomplished by moving the teapot closer to the camera, and :vas thus accompanied by some changes in lighting. This second teapot is scaled larger in size by a factor of 1.36, which is just less than $\sqrt{2}$. The distance and orientation for each P-Path link in this second teapot levels 12 through 7 is shown in table 2 below.

The highest level M-node in this second teapot occurs at level 9. The fact that this $M-$ node is one level higher than the highesi level M-node for teapot 1 confirms that this second teapot is approximately $\sqrt{2}$ larger than the first teapot.

The correspondence of the highest level M-nodes from these two teapots gives an estimatc of the alignment of the two teapots as well as the scaling. The correspondence tcils us the position at which the first teapot, scaled by $\sqrt{2}$ in size will match this second teapot. The tolcrance of the initial position alignment is \pm the sample rate at the level of the $\mathrm{M}$-node in the data image. If this second teapot is designated as the data inage, then the sample rate at level 9 determines the tolerance. The positioning tolerance at level 9 is $\pm 8 \sqrt{2}$ pixcls.

The tolerance of the size scaling is less than $\pm \sqrt{2}$. The correspondence of the highest level M-nodes provides an estimate of the size scaling factor which is a power of $\sqrt{2}$. Such an estimate is sufficient to 


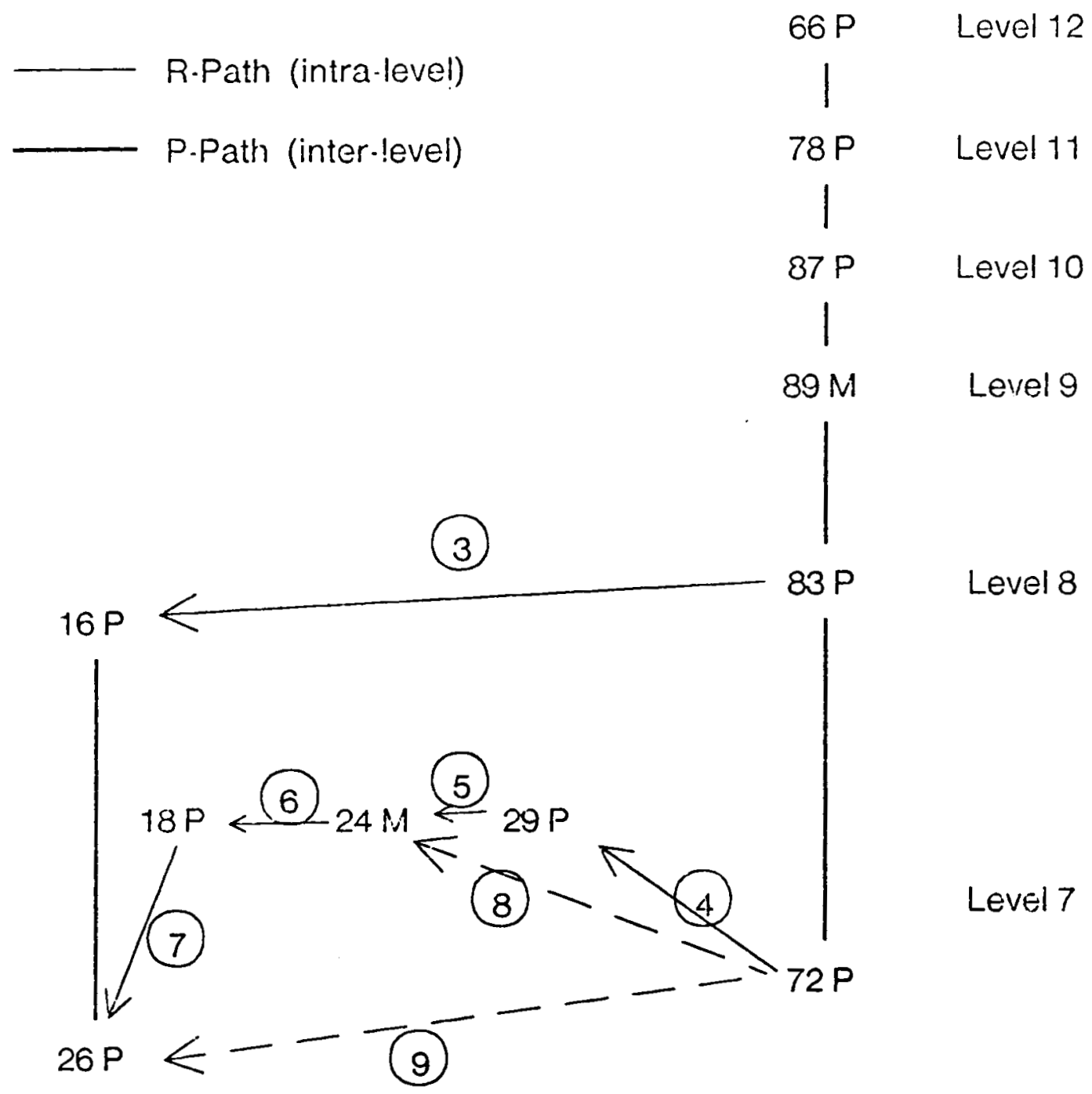

Figure 10: P-nodes and P-Paths for Levels 12 to 7

of Second Teapot (Scaled Larger in Sizc by 1.36) 


\begin{tabular}{llllll} 
R-Path & I.evel & $\mathrm{dx}$ & $\mathrm{dv}$ & $\mathrm{D}$ & $\theta$ \\
\hline 3 & 8 & $-7.5 \sqrt{2}$ & $1.5 \sqrt{2}$ & 10.81 & $191.3^{\circ}$ \\
4 & 7 & -3.5 & -6.0 & 6.94 & $149.7^{\circ}$ \\
5 & 7 & -4.0 & 1.0 & 4.12 & $194.0^{\circ}$ \\
6 & 7 & -4.5 & 1.0 & 4.61 & $192.0^{\circ}$ \\
7 & 7 & -0.5 & 5.0 & 5.02 & $264.3^{\circ}$ \\
$8(4 \& 5)$ & 7 & -10.0 & -1.5 & 10.11 & $171.5^{\circ}$ \\
$9(+\& 5 \& 6 \& 7)$ & 7 & -15 & 3.5 & 15.4 & $193.1^{\circ}$
\end{tabular}

Table 2: R-Path I.inks for l.evels 8 and 7 of the Second Teapot

(Scaled iarger in Size by 1.36 )

constrain the correspondence process. A more accurate cstimate can be obtained from the correspondence of higher resolution P-nodes and M-nodes.

\subsection{Determining Further Correspondence and Orientation}

The matching process starts by finding the correspondence for the highest level W-nodes. This provides the process with an initial estimates of the size and position of the two forms. The next step is to find the corrsponderice of lower level P-nodes and M-rodes to refine the estimates of relative size and pesition, discover the relative orientations, and discover where one of the forms has been distorted by parallax or other arfects.

I.ct us continue with our example. A P-node for the upper left comer of this second teapot docs not occur. The change in scale from the first teapoc to this sccond teapot was not enough to bring this $P$-node up to level 8. This may also be a result of the slight difference in shading that resulted from moving the teapot with respect to the lights and camera in order to size scale the object. Such errors are a natural result of changing the relative position between the camera and objects. A matching algorithm must tolerate them to be useful. The fact that the P-node of value 16 in level 8 of this second teapot corresponds to the P-node of value 14 in level 7 of the first teapot must be discovered from the position relative to their principal P-nodes and the distance and orientation from the P-node on the principal P-path at the same level.

\begin{tabular}{lllllllll} 
& \multicolumn{9}{c}{ Teapot 1} & \multicolumn{3}{c}{ Teapot 2 } & \multicolumn{2}{c}{ Difference } \\
R-Path & $\mathrm{D}_{1}$ & $\theta_{1}$ & $\mathrm{D}_{2}$ & $\theta_{2}$ & $\theta_{1}-\theta_{2}$ & $\mathrm{D}_{2} / \mathrm{D}_{1}$ & $\mathrm{D}_{2}-\mathrm{D}_{1}$ & $100 \times\left(\mathrm{D}_{2}-\mathrm{D}_{1}\right) / \mathrm{D}_{2}$ \\
\hline 3 & 11.09 & $185^{\circ}$ & 10.8 & $191^{\circ}$ & $-6^{\circ}$ & 0.974 & -0.2 & $-1.8 \%$ \\
4 & 6.3 & $153^{\circ}$ & 6.9 & $148^{\circ}$ & $5^{\circ}$ & 1.095 & 0.6 & $8.7 \%$ \\
5 & 5.1 & $206^{\circ}$ & 4.1 & $194^{\circ}$ & $12^{\circ}$ & 0.804 & 1.0 & $24.4 \%$ \\
6 & 4.2 & $180^{\circ}$ & 4.6 & $192^{\circ}$ & $12^{\circ}$ & 1.09 & 0.4 & $8.7 \%$ \\
7 & 4.6 & $266^{\circ}$ & 5.2 & $264^{\circ}$ & $2^{\circ}$ & 1.13 & -0.6 & $-11.5 \%$ \\
8 & 10.2 & $176^{\circ}$ & 10.1 & $171^{\circ}$ & $5^{\circ}$ & 0.99 & -0.1 & $-1.0 \%$ \\
9 & 14.6 & $195^{\circ}$ & 15.4 & $193^{\circ}$ & $2^{\circ}$ & 1.05 & 0.8 & $5.2 \%$ \\
\multicolumn{2}{l}{ Average Error } & & & & $4.57^{\circ}$ & 1.020 & 0.257 & $4.3 \%$
\end{tabular}

Table 3: Comparison of D and $\theta$ attributes for Teapots 1 and 3 
The values for $D$ and 0 for the link attributes in levels 7 and 6 of teaput 1 are compared to the attributes in the corresponding links from level; 8 and 7 of teapot 2 in table 3. Nll of these links are constrained to begin and end at samples in their respetive levels. Because we are dealing with distances of between 4 and 15 samples at arbitrary angles, there is quantization noise in these attributes. The differences in orientution are shown in the column labeled $\theta_{1}-y_{2}$. Except for link 3, these values show a consistent small rotation in the counter-clockwise dircetion for the links fiom teapot 2. A careful measurement of the angle between the line connecting two lindmarks and the raster line in the two images confinns that the two teapots actually have a relative change in orientation of approximately $3.3^{\circ}$. The actual values of 0 fluctuate more than this due to quantization error from sampling and changes in shading.

The ratio $D_{2} / D_{1}$ show's a factor by which the lengths consistently shift when the teapot is scaled by 1.36 . Because the actual valies of $D_{2}$ aitd $D_{1}$ are restricted to distances between discrete locations, there is sume random error built inte this ratio. Since this shift in scale was enough to drive the corresponding R-paths in this second teapot up to a new level, but less than the $\sqrt{2}=1.41$ scale change between icvels, an average ratio of $D_{2} / D_{1}=1.36 / 1.41=0.96$ was anticipated. In table 3 we see that this average ratio worked out to 1.02. Our conclusion is that quantization noise and changes in shading accounted for most of this difference. The actual differences in length, $D_{2}-D_{1}$, show that the lengths are always :vithin one sample. Except for link 5 , the percentage differences. $\left(D_{2}-D_{1}\right) / D_{2}$ are generally small $(\leq 10 \%)$. The conclusion from this experiment is that the correspondence between $R$-nodes from similar gray-scale forms of different sizes can be found, provided that the manhing wherates variations of the lengths of $R$-paths of up to $25 \%$ and variations in the relative angles of up to $12^{\circ}$.

\section{Comments}

The representation for gray scale shape which is formed by detecting peaks and ridges in a resampled DOLP transform resembles the representation provided by a Medial Axis Transform (WNT) described by Blum [5]. There are, however, several important differences. It is worth while to compare these two representations and examine their similarities and differences.

\subsection{Comparison With Blum's Medial Axis Transform}

The MAT ( or grass-fire transform) is a technique for deriving a spine for a binary shape. The transform is defined as follows: Every point on the boundary of the binary shape simultaneously emits a circular wave. The waves propagate in such a manner that waves do not flow through each other. When waves meet head on, they cancel. The point at which they cancel is marked as a point on the M $\Lambda T$ spine of the shape. By propagating the waves in discrete time units, and kecping track of the time at which waves cancel, the spine may be encoded with the distance to the boundary. An axis occurs inside every concave curve, whether it is inside of a shape or not.

Rosenfeld [27] has shown a fast two pass operator which will implement the grass fire transform. This operator is significant on its own right because it makes possible the matching technique of "Chamfer Matching" [6].

There are at least two fundamentai problems which prevent the spine from a MAT from being uscful for 
describing gray-scale shape. The first of these is that the transform only exists for binary shapes. The second problem. first pointed out by $A$ gin [2], is that a small narrow concavity in the boundary will signiticantly alter the shape of the resulting spine. Similar effects can oceur from many other types of noise patterns. Thus the transform and the spine are very sensitive to noise.

In rontrast, the representation giren by peaks and ridges in a DOI.P transform is a representation for gray scale shape instead of binary shape. The DOLP band-pass filters have a circular positive center lobe which is a best fit to the gray scale pattern when the DOLP value is large. Thus, as with the MAl spine, the DOL.P ridges tend to exist where a circle is a best fit to the pattern. However, the DOLP band-pass filters have a smoothing effect: they are only sensitive to patterns at narrow range of sizes (spatial frequencies). Thus a narrow concavity is described in detail by small DOL.P filters, the concavity has almost no effect on the ridge given by large DOLP filters.

The representation given by peaks and ridges in the DOLP transform has many other propertics which a MAT spine does not have: For example, there is the existence of a largest peak as a landmark for matching, the fact that the representation can be used to guide matching from course resolution to high resolution, and the important property that the configuration of peaks and ridges can be matched when the pattern occurs at any size.

\section{Summary and Conclusion}

The principal topic of this paper is a representation for grey scale shape which is composed of pcaks and ridges in the DOLP transform of an image. Descriptions of the shape of an object which are enceded in this representation may be matched efficiently despite changes in size, orientation or position by the object. Such descriptions can also be matched when the object is blurry or noisy.

The definition of the DOLP Transform was presented, and the DOLP Transform was shown to be reversible. $A$ fast algorithm for computing the DOLP Transform based on the techniques of resampling and cascaded convolution with expansion was then described. This fast algorithm is described in greater detail in [14]. This section concluded with an example of the DOLP transform of an inage which contains a tcapot.

A representation for gray-scale form based on the peaks and ridges in a DOLP transform was then described. This representation is composed of four types of symbols: $\{M, P, L, R\}$. The symbols $R$ and $P$ (Ridge and Peak) are detccted within cach DOLP band-pass image. R-nodes are samples which are local positive maxima or negative minima among three contiguous DOLP samples in any of the four possible directions. P-nodes are samples which are local positive maxima or negative minima in all four directions. $P$-nodes within the same form in a band-pass level are connected by a path of largest $R$-nodes, called an $\mathrm{R}$-path (or ridge). An R-path is formed by having each $\mathrm{R}$-node make a pointer to members of its local neighborhood which are also $R$-nodes and local maxima within a linear list of the neighborhood. P-nodes are connected with nearby P-nodes at adjacent band-pass levels to form P-paths. The skeleton of the description of a form is a tree composed of P-paths.

The DOLP values along each P-path rise monotonically to a maximum in magnitude and then decrease. The maximum magnitude DOL.P sample along a P-path is marked as an M-node. M-nodes serve as landmarks for matching, and provide an estimate of the position and orientation of a form in an image. If the 
values along an $R$-path are compared to the values along the R-paths at nearby lucations in ikljacent bandpass images, an R-path of largest DOLP samples can be detected. These somples are marked as L-nedes, and the these nodes form an I.-path. I.-paths besin and end at M-nodes and describe clongated forms. Thus, descriptions in this representation have the structure of a trec composed of P-paths, with a distinguished M-node along each. The P-nodes in each level are connected by R-paths, and the M-nodes are connected by L-paths which can travel among as well as within the levels.

The teapot image was used to illustrate the construction of a description in this represcatation. In this iliustration, the R-nodes and P-nudes from band-pass level 7 from the DOLP transform of the teapot and the pointers between these $R$-nodes were displayed.

The final section of the paper presented a description and examples of the problem of determining the correspondence between the M-nodes and P-nodes in two descriptions of the same object. A description of a second teapot image, in which the teapot had been moved so as to be scalcd larger by 1.36 , was used to illustrate the principles of matching such descriptions. In both teapot imagcs, the P-paths, R-paths and $M$-nodes from the coarsest resolution band-pass inages were presented. Matching to determine the correspondence of $L$-paths was not described in this paper. Such matching is described in [13].

The te?pot matching examples first illustrated the correspondence of the coarsest iesolution $M$-nodes in the two descriptions. This correspondence provides an estimate of the position and size at which the two teapot description best match. The principle that P-nodes in two descriptions can only correspond if the P-nodes doove them correspond was also illustrated. An example was then provided for the use of the lengths and directions of the R-patiss that connect P-nodes at each level to further determine correspondence when new $P$-paths are introciuced and the orientation has not been determined.

This example addresses snly a small part of the general problem of matching descriptions of objects. The problem of matching two descriptions of an object with large differences in image plane orientation was not illustrated. An example of such matching is provided in [13]. The more difficult problems of matching in the presence of motion of either the camcra or the object was not discussed. Such matching must be robust enough to accommodate the changes in two-dimensional shape that occur with a changing threc-dimensional viewing angle. Similarly, the problens of forming and matching to a prototype for a class of objccts was not discussed. We believe that this representation will provide a powerful structural pattern recognition technique for recognizing objects in two-dimensional domain and for dynamically constructing a threedimensional model of a three-dimensional scene.

\section{Acknowledgements:}

We would like dedicate this paper to Frank Quick, who first posed many of the questions which led us to develop the DOLP transform and this representation. We would also like to thank the Dr. Raj Reddy and the Carnegie-Mellon University Computer Science Department who provided computer time and disc space for this research. Their support permitted this research to continuc at a critical time during which there was no funding. We would also like to thank Richard Stern, Takeo Kanade and Art Sanderson who generously provided the intellectual critiques and emotional support during the course of this research. Finally, we would like to thank the Westinghouse Electric Corporation which has recently made it possible to continue this rescarch from the point described here. 


\section{References}

[1] Abramatic, J. F. and O. D. Faugcras.

Scquential Convolution Techniques for Image Filtering.

IEEE Trans. on Acous. Speech and Signal Processing ASSP-30(1): 1-10, F-cbuary, 1982.

[2] Agin, G. J.

Representation and Description of Cunved Objects.

PhD thesis, Stanford University, 1971.

[3] Aho, Nired V., John F. Hopcroft, and Jeffery D. Ullman.

Computer Science and Information Processing: The Design and Analysis of Computer Alsorithms.

Addison Wesley, Reading Massachusetts, 1974.

[4] Binford, Thomas O.

Survey of Model-Based Image Analysis Systems.

Robolics Research 1(1):18-64, Spring, 1982.

[5] Blum.

A Transformation for Extracting New Descriptors of Shape.

In Models for the Percepiion of Speech and Visual Form, . MIT Press, Canbridge, 1967.

[6] Barrow, H. G., J. M. Tcnenbaum, R.C. Bolles, and H.C. Wolf.

Parametric Correspondence and Chamfer Malching: Two New Techniques for Image Matching.

Technical Note 153, SRI International, 1978.

[7] Burt, Peter J.

Fast, Hierarchical Correlations with Gaussian-Like Kernels.

Techuical Report TR-860, Computer Vision Laboratory, University of Maryland, January, 1980.

[8] Burt, Peter J.

Fast Filter Transforms for Image Processing.

Computer Graphics and Image Processing 16:20-51, 1981.

[9] Campbell, F. W.

The Trunsmission of Spatial Information through the Visual System.

MIT Press, 1974, .

[10] Campbell, F. W. and J. G. Robson.

Applications of Fourier Analysis to the Visibility of Gratings.

Journal of Physiology :551-566, 1971. 
[11] Crowlcy, J. L. and A. C. Parker.

The Analysis. Synthesis, and Evaltation of Local Measures for Discrimination and Segmentation of

Textured Regions.

In Conference on Pallern Recognition and Image Processing, pages 372-378. IEEt: Computer Socicty, June, 1978.

[12] Crowley, J. I.. and A. C. Parker. Transfer Function Analysis of Picture Processing Operators.

In Robert M. Haralick and J. C. Sinron (editor), Issues In Digital hinage Processing, chapter 1, , pages 3-30. Sijthort \& Noordhoff, 1980.

[13] Crowlcy, James L:

A Representation for Visual Information.

PhD thesis, Carnegie-Mcllon University, November, 1981.

[14] Crowley, J. L. and R. M. Stern.

Fast Complitation of the Difference of Low-Pass 'Transform.

Submitted to ihe IEEE Trans. on P.A.M.I. , 1983.

[15] Hall, E. L., Rouge, Lt. J. and Wong, R. Y.

Hierarchical Search for Image Matching.

In Proc. 1976 IEEE Conf. on Decision and Control, pages 791-796. IEEE, December, 1976.

[16] Hanson, A. R. and E. M. Riscman.

Computer Vision Systems.

Academic Press, New York, 1978, pages 758-768.

[17] Kelly, M. D.

Fdge Detection in Computers by Computer Using Planning.

In B. Meltzer and D. Mitchic (cditor), Machine Intelligence, . Halsted Press, 1971.

[18] Marr, D. and Poggio, T.

A Computational Theory of Human Vision.

Proc. R. Soc. Lond B , 1979.

[19] Marr, D. L., and Hildreth, E.

Theory of Edge Detection.

Proc. R. Soc. Lond. B. 207:187-217, 1980.

[20] Marr, David.

Vision

W. H. Frecman and Co., San Francisco, 1982. 
21] Morarce, H. P.

Obstacle Awidance and Navigation in the Real World by a Seeing Robot Rover.

Plil thesis, Stanford University, September, 1980.

[22] Nyquist, [1.

Certain Factors Affecting Tolegraph Specd.

Bell Systems Tech Joumal 3(2):324-346, April, 1924.

[23] Oppenticin, A. V. and Schater, R. W.

Digital Signal Processing.

Prentice-Hall inc., Englewood Cliffs, N. J., 1975.

[24] Palcy R.F.A.C. and N. Wiener.

Fourier Tiansforms in the Complex Domain.

American Mathematical Socicty Coloquium, 19, Now York, 1934.

[25] Papoulis, $\lambda$.

Systems Sciences: Systems and Transforms with Applications in Optics.

McGraw-Hill, New York, 1968.

[26] Pratt, Wiiiiam K.

Digital Image Processing.

John Wilcy ả Sons, 1978.

page 322.

[27] Rosenfeld, A. and J. L. Pflatz.

Distance Functions on Digital Pictures.

Pattern Recognition !(1):33-62, July, 1968.

[28] Rosenfeld, A. and Vanderbrug, G. J.

Coarse-Finc Template Matching.

IEEE Trans. on Man, Systems, and Cybernetics SMC-7(2):104-107, Fcb., 1977.

[29] Sachs, M. , J. Nachmias, and J. G. Robson.

Spatial-Frequency Channels in Human Vision.

Journal of the Optical Society of America 61:1176-1186, 1971.

[30] Tanimoto, S. L. and T. Pavlidis.

A Hicrarchical Data Structure for Picture Processing.

Computer Graphics and Image Processing 4(2):165-174, June, 1975.

[31] Thomas, J. P.

Spatial Resolution and Spatial Interaction.

Academic Press, New York, 1975, . 
[32] Uhr, L.

Layered "Recognition Cone" Networks That Preprocess, Classify, and Describe. IEEE Trans on Computers 21(2):758-768, 1972. 
